\title{
Efecto del conservante azidiol sobre la detección por cultivo microbiológico de bacterias causantes de mastitis bovina ${ }^{1}$
}

\section{ARTÍCULO DE INVESTIGACIÓN}

Giovanny Torres-Lindarte (iD 1,2, Juana Vidal-Arboleda (iD 2,3 , Arley CaraballoGuzmán (D) ${ }^{1}$, Karen Vargas-Hoyos (D) ${ }^{2}$, Martha Olivera-Angel (i] ${ }^{1,2,3}$

1 Instituto Colombiano de Medicina Tropical, Universidad CES. Sabaneta (Antioquia), Colombia.

2 Grupo Biogénesis, Universidad de Antioquia. Medellín, Colombia. 3 Unidad de Diagnóstico, Facultad de Ciencias Agrarias, Universidad de Antioquia. Medellín, Colombia.

\section{martha.olivera@udea.edu.co}

Recibido: 8 de Mayo de 2017 y aprobado: 17 de Agosto de 2017, Actualizado: 12 de Septiembre de 2017

DOI: 10.17151/vetzo.2017.11.2.8

RESUMEN: El azidiol es un compuesto químico con efecto bacteriostático recomendado para la conservación de muestras de leche cruda destinadas tanto al análisis composicional como higiénico-sanitario. No obstante, su uso para conservar muestras que serán empleadas en el diagnóstico de patógenos causantes de mastitis no ha sido claramente documentado. El objetivo del trabajo fue evaluar el efecto del conservante azidiol sobre la detección por cultivo microbiológico de bacterias causantes de mastitis. Muestras de leche, con y sin azidiol, fueron inoculadas con dos concentraciones bacterianas de cada cepa incluida (Staphylococcus aureus,Staphylococcus epidermidis, Streptococcus agalactiae, Escherichia coli). Los resultados demostraron que las bacterias de los géneros Staphylococcus yStreptococcus fueron aisladas de las muestras que contenían azidiol 24 horas después de la inoculación con ambas concentraciones bacterianas de ensayo; aunque, ningún cultivo superó el número de unidades formadoras de colonia inoculadas al inicio. $\boldsymbol{S}$. agalactiae fue la única bacteria que se pudo aislar a las 48 y 72 horas de incubación de las muestras inoculadas con las dos concentraciones evaluadas y que contenían el preservante; mientras que $\boldsymbol{E}$. coli no se aisló de ninguna muestra con azidiol. En las muestras control (sin conservante) se lograron aislar las cuatro cepas en los tres tiempos de incubación. En conclusión, las muestras conservadas con azidiol pueden ser usadas para el diagnóstico de bacterias Gram positivas causantes de mastitis dentro de las 24 horas posteriores a la recolección, pero no es recomendado para conservar muestras de leche que serán procesadas después de este tiempo ni para el diagnóstico de bacterias Gram negativas.

Palabras clave: análisis bacteriológico, Escherichia coli, preservación de muestras, refrigeración, Staphylococcus, Streptococcus. 


\title{
Effect of the preservative azidiol on the detection of mastitis-causing bacteria by microbiological culture
}

\begin{abstract}
Azidiol is a chemical compound with bacteriostatic effect recommended for preserving raw milk samples, which will be used for compositional and hygienic-sanitary analyses. However, its use to preserve milk samples which will be used in the diagnosis of mastitis-causing pathogens, has not been clearly documented. The objective of this study was to evaluate the effect of azidiol on the microbiological culture detection of mastitis-causing bacteria. Milk samples, with and without azidiol, were inoculated with two bacterial concentrations from each strain included (Staphylococcus aureus, Staphylococcus epidermidis, Streptococcus agalactiae, Escherichia coli). The results demonstrated that bacteria of Streptococcus and Staphylococcus genera, were isolated from the samples containing azidiol 24 hours after the inoculation with both bacterial concentrations, although no culture exceeded the number colony forming units inoculated at the beginning. S. agalactiae was the only bacterium that could be isolated at 48 and 72 hours of incubation of the samples inoculated with both bacterial concentrations and that contained the preservative, while $\boldsymbol{E}$. coli was not isolated from any sample with azidiol. In the control samples (without preservative) the four strains were isolated during the three incubation times. In conclusion, the milk samples preserved with azidiol can be used on the diagnosis of mastitis-causing Gram-positive bacteria within 24 hours after the collection of milk samples, but this is not recommended neither for preserving milk samples which will be processed after this time, nor in the diagnosis of the mastitis-causing Gram-negative bacteria.
\end{abstract}

Key words: bacteriological analysis, Escherichia coli, refrigeration, samples preservation, Staphylococcus, Streptococcus.

\section{Introducción}

La mastitis bovina es un proceso inflamatorio de la glándula mamaria, y es considerada la enfermedad que más pérdidas económicas le genera a los productores de leche a nivel mundial. En el estudio realizado por Kosaibati \& Esslemont (1997) estimaron que la mastitis representaba el 38\% de los costos directos totales de las enfermedades de toda la producción en el Reino Unido (Kossaibati \& Esslemont, 1997; Philpot \& Nickerson, 2000; Shim et al., 2004).

Alrededor de 150 microorganismos se han relacionado con la mastitis de tipo infeccioso; sin embargo, son los cocos Gram positivos de los géneros Staphylococcus yStreptococcus los responsables de la mayoría de casos (Fu et al., 2013). Aunque, en países como Canadá e Inglaterra los bacilos Gram negativos como Escherichia colipresentan prevalencias significativas (Bradley et al., 2007; Olde et al., 2008).

El incremento de la resistencia microbiana a los antibióticos, la variación en los Recuentos de Células Somáticas (RCS) que se pueden presentar en algunas mastitis y el hecho de que no todas las infecciones requieren tratamientos antibióticos, han convertido el cultivo microbiológico en una herramienta fundamental para los 
programas de control de mastitis. El cultivo permite aislar e identificar el patógeno responsable de la infección, determinar la susceptibilidad a los antibióticos y verificar la eliminación del microorganismo después del tratamiento aplicado al animal (Britten, 2012).

Para un acertado diagnóstico microbiológico por cultivo es indispensable garantizar la integridad de la muestra desde su recolección hasta su procesamiento en el laboratorio, pues la pérdida de viabilidad o inhibición de los microorganismos, así como la contaminación, conllevarán a resultados no confiables (falsos negativos o falsos positivos). Uno de los factores que puede afectar la viabilidad microbiana es el método usado para la preservación de la muestra (Almeida et al., 2016). El National Mastitis Council (NMC) recomienda la refrigeración o la congelación como mecanismos de conservación de las muestras de leche destinadas para el diagnóstico de patógenos causantes de mastitis, debido a que logran mantener los microorganismos vivos por varios días o semanas, respectivamente (National Mastitis Council, 2004). Sin embargo, dadas las condiciones a nivel rural en Colombia no siempre se puede contar con un sistema que permita mantener las muestras refrigeradas después de recolectadas; incluso, en los casos en los cuales es posible refrigerarlas, los retrasos durante el transporte pueden ocasionar que estas lleguen al laboratorio con temperaturas superiores a los $10^{\circ} \mathrm{C}$ (Almeida et al., 2016).

Diferentes autores han estudiado el efecto de algunos conservantes sobre la composición, el RCS y el Recuento Total de Bacterias (RTB) en leche cruda (Llopis et al., 2013). La refrigeración, el bronopol y el azidiol son algunos de los conservantes de muestras de leche cruda empleados con mayor frecuencia en la actualidad (Elizondo et al., 2007; Cassoli et al., 2010). Por ejemplo, en España el uso de azidiol cada vez es más frecuente en los laboratorios de análisis de leche (Llopis et al., 2013). En el trabajo realizado por Cassoli et al. (2010) se demostró que la leche cruda refrigerada a $7^{\circ} \mathrm{C}$ más azidiol o bronopol puede ser analizada hasta siete días después de recolectada; mientras que Leite (2006) encontró que las muestras con azidiol y almacenadas a $10^{\circ} \mathrm{C}$ se conservaban hasta por 10 días.

El uso del azidiol para conservar muestras que serán empleadas en el diagnóstico de patógenos causantes de mastitis no ha sido claramente documentado, pues se ha descrito que este puede afectar el crecimiento de bacterias como Staphylococcus y E. coli, importantes patógenos causantes de mastitis (Elizondo et al., 2007). No obstante, resultados obtenidos en dos laboratorios de diagnóstico de mastitis ubicados en Antioquia (Colombia) evidenciaron el aislamiento de Staphylococcus aureus yStreptococcus uberis en muestras de leche recolectadas de animales con mastitis y conservadas con azidiol (datos no publicados); de manera que podría ser considerado como una posible alternativa de conservación para los patógenos bacterianos presentes en las muestras de leche.

Por lo tanto, el objetivo del trabajo fue evaluar el efecto del conservante azidiol sobre la detección por cultivo microbiológico de bacterias causantes de mastitis. 


\section{Materiales y Métodos}

\section{Preparación de cepas evaluadas}

Se evaluaron cuatro cepas de bacterias causantes de mastitis, pertenecientes a la colección de American Type Culture Collection (ATCC) (Tabla 1). Inicialmente, se preparó una suspensión bacteriana de cada una de las cepas a una concentración entre 1,5-4,5 x $10^{8}$ Unidades Formadoras de Colonias (UFC) por mililitro (ml). Cada suspensión inicial fue diluida hasta alcanzar las concentraciones 1,5-4,5 x 106 UFC/ml y 1,5-4,5 x $10^{5}$, las que en adelante se denominarán suspensiones de prueba 1 (SP1) y 2 (SP2), respectivamente. Las concentraciones se confirmaron mediante la siembra y recuento en placa.

Tabla 1. Cepas de referencia empleadas en el estudio

\begin{tabular}{cc}
\hline Cepa & Referencia ATCC \\
\hline Staphylococcus aureus & 25923 \\
\hline Streptococcus agalactiae & 27956 \\
\hline Staphylococcus epidermidis (SCN) & 12228 \\
\hline Escherichia coli & 25922 \\
\hline SCN: Staphylococcus Coagulasa Negativo.
\end{tabular}

\section{Preparación de las muestras de leche}

Como muestra se utilizó leche entera Ultra High Temperature (UHT) marca Colanta ${ }^{\circledR}$. Para la evaluación de cada cepa, se prepararon ocho frascos de recolección de muestras de leche estériles (ECSI S.A.S., referencia F250528), cuatro con azidiol (LABORCLIN ${ }^{\circledR}$, referencia 610903) y cuatro sin este. Una vez establecidas las suspensiones de prueba A y B, a cada frasco se le adicionaron $40 \mathrm{ml}$ de muestra; los que contenían el azidiol se mezclaron hasta que los comprimidos se disolvieron totalmente. Cuatro de los ocho frascos, dos con azidiol y dos sin este, fueron empleados para evaluar la concentración de ensayo 1 (C1), la cual fue estimada entre 1-5 UFC/10 $\mu \mathrm{L}$; los cuatro restantes para la concentración de ensayo 2 (C2), estimada entre 15-45 UFC/10 $\mu \mathrm{L}$. Con estas concentraciones se garantizaba muestras con bajos recuentos bacterianos, condición que pondría a prueba la capacidad del azidiol para preservar la viabilidad de las cepas hasta su posterior recuperación.

En total se prepararon 34 muestras; 32 para las inoculaciones, ocho por cada cepa evaluada; los dos últimos contenían únicamente leche UHT y se emplearon como control de esterilidad de la leche.

\section{Inoculación de las muestras con las suspensiones de prueba}

Una vez las muestras estuvieron preparadas, se inocularon con las suspensiones de prueba SP1 y SP2. Las dos muestras que contenían azidiol y dos sin este fueron inoculadas con $40 \mu \mathrm{L}$ de la SP2 para obtener la C1. Las otras cuatro muestras fueron inoculadas con $40 \mu \mathrm{L}$ de la SP1 para obtener la C2. El procedimiento se repitió para 
cada cepa. Las muestras se incubaron durante 24, 48 y 72 horas a temperatura ambiente $\left(20^{\circ} \mathrm{C}-23^{\circ} \mathrm{C}\right)$; condición que simulaba el transporte de estas sin refrigeración.

Los controles de inoculación se realizaron a partir de las muestras que no contenían azidiol mediante la siembra y recuento en agar sangre.

\section{Siembra de las muestras inoculadas}

Después de cumplidos los tiempos de incubación (24, 48 y 72 horas), se sembraron 10 $\mu \mathrm{L}$ en agar sangre de cada una de las muestras inoculadas con cocos Gram positivos ( $\boldsymbol{S}$. aureus, $\boldsymbol{S}$. agalactiae y $\boldsymbol{S}$. epidermidis) y de los controles de esterilidad. Por otra parte, se sembraron en agar MacConkey $10 \mu \mathrm{L}$ de las muestras inoculadas con el bacilo Gram negativo (E. coli) (National Mastitis Council, 2004). Se realizaron las diluciones necesarias para garantizar el adecuado recuento de las UFC por placa. Los medios se incubaron a $37^{\circ} \mathrm{C}$ hasta por 72 horas, con recuentos cada 24 horas.

Todas las muestras y controles se sembraron por duplicado y el ensayo se realizó tres veces en momentos diferentes.

\section{Análisis de los datos}

Los recuentos bacterianos obtenidos durante los tres ensayos, expresados en UFC, fueron promediados, almacenados y procesados en Microsoft Excel 2013 con previa revisión de los datos. Además, con el fin de comparar el efecto del tratamiento con azidiol sobre las UFC/10 $\mu \mathrm{L}$, se realizó el test no paramétrico U de Mann-Whitney a las 24, 48 y 72 horas para todas las especies bacterianas y concentraciones del producto.

\section{Resultados y Discusión}

\section{Recuentos de los controles de inoculación}

Los promedios de los recuentos de las cepas obtenidos inicialmente, correspondientes a los controles de inoculación de las muestras, estuvieron entre 1 y 3 UFC/10 $\mu \mathrm{L}$ para la C1 y entre 15 y $29 \mathrm{UFC} / 10 \mu \mathrm{L}$ para la C2, demostrando que todas las muestras fueron inoculadas con las concentraciones estimadas en la metodología (Tabla 2).

Tabla 2. Recuentos controles de inoculación de muestras

\begin{tabular}{ccc}
\hline \multirow{2}{*}{ Cepa } & C1 & C2 \\
\cline { 2 - 3 } & UFC $/ 10 \mu \mathrm{L}$ & UFC $/ 10 \mu \mathrm{L}$ \\
\hline S. aureus & 2 & 28 \\
\hline S. agalactiae & 3 & 25 \\
\hline S. epidermidis & 1 & 15 \\
\hline E. coli & 2 & 29 \\
\hline
\end{tabular}

C1: Concentración 1; C2: Concentración $2 ; \mu \mathrm{L}$ : microlitro. 


\section{Recuentos de las cepas durante el ensayo}

Los promedios obtenidos de los tres ensayos realizados con la cepa de $\boldsymbol{S}$. aureus se presentan en la Tabla 3. Se observó en ambas concentraciones un crecimiento exponencial durante los tiempos de incubación establecidos. Por el contrario, los recuentos a las 24 horas de incubación en las muestras con azidiol no superaron las concentraciones de inoculación inicial, alcanzaron máximo $2 \mathrm{UFC} / 10 \mu \mathrm{L}$ en la C1 y 8 $\mathrm{UFC} / 10 \mu \mathrm{L}$ en la C2. A las 48 horas solo se recuperó 2 UFC/10 $\mu \mathrm{L}$ en la C2 y a las 72 horas no se obtuvo crecimiento bacteriano (Tabla 4).

Para la cepa de $\boldsymbol{S}$. agalactiae evaluada, los promedios obtenidos con las dos concentraciones bacterianas en las muestras sin azidiol presentaron un comportamiento similar al observado con la cepa de $\boldsymbol{S}$. aureus (Tabla 3). Los recuentos en el ensayo con azidiol presentaron a las 24 horas un promedio de 3 UFC/10 $\mu \mathrm{L}$ en la C1 y 21 UFC/10 $\mu \mathrm{L}$ en la C2, también dentro de los recuentos estimados para las inoculaciones iniciales; estos recuentos decrecieron durante todo el ensayo obteniendo concentraciones finales (72 horas) de $1 \mathrm{UFC} / 10 \mu \mathrm{L}$ y $6 \mathrm{UFC} / 10 \mu \mathrm{L}$, respectivamente (Tabla 4).

La cepa de $\boldsymbol{S}$. epidermidis evaluada presentó promedios de crecimiento menores a los observados con las demás cepas en las muestras sin azidiol (Tabla 3). Los resultados obtenidos al utilizar azidiol como conservante mostraron recuentos iniciales (24 horas) de $1 \mathrm{UFC} / 10 \mu \mathrm{L}$ en la C1 y $11 \mathrm{UFC} / 10 \mu \mathrm{L}$ en la C2, pero llegaron a $0 \mathrm{UFC} / 10 \mu \mathrm{L}$ a las 48 horas y se mantuvieron negativos hasta las 72 horas (Tabla 4).

E. coli fue la cepa evaluada que presentó mayor crecimiento a las 24 horas para cada una de las concentraciones bacterianas en las muestras sin azidiol (Tabla 3). En el ensayo con azidiol no se obtuvo crecimiento en ninguno de los tiempos de incubación evaluados (Tabla 4).

En el análisis estadístico se observaron diferencias estadísticamente significativas entre los grupos de tratamiento para cada una de la mediciones a las 24 ( $\mathrm{P}=0,0027), 48$ $(\mathrm{P}=0,0006)$ y 72 horas $(\mathrm{P}=0,0006)$, respectivamente. En general, para cada una de las mediciones se observaron valores mayores de UFC/10 $\mu \mathrm{L}$ en el grupo no tratado que en el tratado con azidiol.

El uso de azidiol como conservante ha sido recomendado por algunos autores para el análisis químico, físico y bacteriológico de la leche (Elizondo et al., 2007; Martins et al., 2009; Sierra et al., 2009; De Garnica et al., 2011; Llopis et al., 2013; Peixoto et al., 2016), sin embargo, los resultados obtenidos durante el ensayo confirman lo reportado por Benda (1995) y Elizondo et al. (2007), quienes afirman que bacterias como $\boldsymbol{E}$. coliy $S$. aureus son sensibles al conservante y su desarrollo se puede ver afectado. Los cocos Gram positivos incluidos, $\boldsymbol{S}$. aureus, $\boldsymbol{S}$. epidermidis y $\boldsymbol{S}$. agalactiae, se aislaron de las muestras que contenían azidiol 24 horas después de la inoculación con las dos concentraciones bacterianas evaluadas; aunque, ningún cultivo superó el número de UFC inoculadas al inicio. $\boldsymbol{S}$. agalactiae fue la única bacteria que se pudo aislar a las 48 y 72 horas de incubación de las muestras que contenían el preservante e inoculadas con las dos concentraciones bacterianas; mientras que $\boldsymbol{E}$. coli no se aisló de ninguna muestra con azidiol. En todas las muestras control (sin conservante) inoculadas con las dos 
concentraciones de cada cepa se obtuvo crecimiento en los tres tiempos de incubación. Lo anterior indica que el azidiol afectó el desarrollo bacteriano durante el periodo de estudio; no obstante, fue posible aislar los cocos Gram positivos 24 horas después de la inoculación, por lo que las muestras conservadas con azidiol pueden ser usadas para el diagnóstico de este tipo de bacterias dentro de las 24 horas posteriores a la recolección, pero no es recomendado para preservar muestras de leche que serán procesadas después de este tiempo, ni para el diagnóstico de bacterias Gram negativas (Neder et al., 2014). Aunque, el azidiol es uno de los conservantes recomendados para muestras que serán empleadas para el RTB por métodos automatizados (Sierra et al., 2009; Almeida et al., 2016), debería ser cuidadosamente validado en cada laboratorio, pues Elizondo et al. (2007) demostraron el efecto de la concentración del cloranfenicol (Cl) y la azida sódica (As) sobre el RTB en leche de ovejas realizado con un método automatizado, observando que los recuentos más cercanos a los controles (muestras sin azidiol) correspondían a las muestras que solo contenían la mitad de la concentración de Cl y As de la formulación estándar del azidiol empleada (0,15\% de $\mathrm{Cl}$ y 3,6\% de As) (Elizondo et al., 2007).

Diferentes autores han demostrado el efecto de la temperatura sobre el recuento bacteriano, incluso, cuando se emplea en conjunto con otros conservantes, tales como el azidiol (Leite, 2006; Cassoli et al., 2010). En un estudio reciente realizado por Almeida et al. (2016) se evidenció que las muestras almacenadas a $11^{\circ} \mathrm{C}$ para el $\mathrm{RTB}$ se conservaron hasta por 16 días; lo que difiere de los resultados obtenidos en otros estudios, en los cuales el tiempo máximo de conservación reportado estuvo entre 7 y 10 días (Leite, 2006; Souza et al., 2006; Cassoli et al., 2010). Almeida et al. (2016) también observaron que el uso del azidiol en las muestras conservadas a temperatura ambiente $\left(25^{\circ} \mathrm{C}\right)$ no fue suficiente para prevenir el crecimiento de las bacterias, pues a los 16 días estas habían superado el recuento de 7 LogUFC/ml (Almeida et al., 2016). La multiplicación acelerada de las bacterias es una condición que impacta negativamente sobre su propia viabilidad, debido a la disminución de nutrientes y a la acumulación de metabolitos nocivos en el medio (Neidhardt et al., 1990), de modo que a mayor temperatura de almacenamiento menor será el tiempo de conservación de la muestra. Los resultados observados en las muestras control (sin conservante) para las concentraciones y bacterias evaluadas, demostraron que la temperatura ambiente $\left(20^{\circ} \mathrm{C}\right.$ a $23^{\circ} \mathrm{C}$ ) a la cual se almacenaron las muestras durante su seguimiento (72 horas), no influyó sobre la viabilidad de las bacterias inoculadas en la leche con preservante; por el contrario, favoreció el crecimiento de las cepas en las muestras control, lo que garantizó su recuperación por cultivo bacteriológico.

Tabla 3. Recuentos promedio de las cepas evaluadas en muestras de leche sin preservante

\begin{tabular}{|c|c|c|c|c|c|c|}
\hline \multirow[t]{2}{*}{ Cepa } & \multicolumn{3}{|c|}{$\begin{array}{l}\text { Recuento en C1 } \\
\text { (UFC/10 } \mu \mathrm{L} \text { ) }\end{array}$} & \multicolumn{3}{|c|}{$\begin{array}{l}\text { Recuento en C2 } \\
\text { (UFC/10 } \mu \mathrm{L} \text { ) }\end{array}$} \\
\hline & $24 \mathrm{~h}$ & $48 \mathrm{~h}$ & $72 \mathrm{~h}$ & $24 \mathrm{~h}$ & $48 \mathrm{~h}$ & $72 \mathrm{~h}$ \\
\hline S. aureus & 2800 & 700000 & 2800000 & 21000 & 1500000 & 4100000 \\
\hline S. agalactiae & 6800 & 300000 & 2100000 & 33000 & 1800000 & 3000000 \\
\hline S. epidermidis & 3 & 470 & 4500 & 30 & 1200 & 3100 \\
\hline E. coli & 21000 & 4200000 & 5000000 & 110000 & 4200000 & 6400000 \\
\hline
\end{tabular}


Tabla 4. Recuentos promedio de las cepas evaluadas en muestras de leche con preservante

\begin{tabular}{|c|c|c|c|c|c|c|}
\hline \multirow[t]{2}{*}{ Cepa } & \multicolumn{3}{|c|}{$\begin{array}{l}\text { Recuento en C1 } \\
\text { (UFC/10 } \mu \mathrm{L})\end{array}$} & \multicolumn{3}{|c|}{$\begin{array}{l}\text { Recuento en C2 } \\
\text { (UFC/10 } \mu \mathrm{L} \text { ) }\end{array}$} \\
\hline & $24 \mathrm{~h}$ & $48 \mathrm{~h}$ & $72 \mathrm{~h}$ & $24 \mathrm{~h}$ & $48 \mathrm{~h}$ & $72 \mathrm{~h}$ \\
\hline S. aureus & 2 & 0 & 0 & 8 & 2 & 0 \\
\hline S. agalactiae & 3 & 2 & 1 & 21 & 5 & 6 \\
\hline S. epidermidis & 1 & 0 & 0 & 11 & 0 & 0 \\
\hline E. coli & 0 & 0 & 0 & 0 & 0 & 0 \\
\hline
\end{tabular}

$\mathrm{C} 1$ : Concentración $1 ; \mathrm{C} 2$ : Concentración $2 ; \mu \mathrm{L}$ : microlitro; h: hora; ${ }^{0} 0^{*}$ : representa que no se obtuvo crecimiento.

\section{Conclusión}

El azidiol afectó negativamente el crecimiento de las bacterias evaluadas bajo el tiempo y las concentraciones bacterianas empleadas en el estudio; sin embargo, en el caso de los cocos Gram positivos fue posible recuperarlos a las 24 horas después de la inoculación, de manera que las muestras conservadas con azidiol pueden ser usadas para el diagnóstico de bacterias Gram positivas causantes de mastitis dentro de las 24 horas posteriores a la recolección, pero no es recomendado para conservar muestras de leche que serán procesadas después de este tiempo, ni para el diagnóstico de bacterias Gram negativas.

\section{Agradecimiento}

A la Universidad de Antioquia y al Laboratorio de Microbiología Industrial del Instituto Colombiano de Medicina Tropical - Universidad CES, por facilitar los recursos económicos y técnicos requeridos para la ejecución del estudio.

\section{Referencias bibliográficas}

- Almeida, T.; Neves, R.; Arnhold, E. et al. Efeito da temperatura e do tempo de armazenamento de amostras de leite cru nos resultados das análises eletrônicas.Arquivo Brasileiro de Medicina Veterinária e Zootecnia, v.68, n.5, p.1316-1324, 2016.

- Benda, J. The effect of some preservatives on natural microflora in milk samples.Veterinarni Medicina, v.40, n.11, 1995. 
- Bradley, A.; Leach, K.; Breen, J. et al. Survey of the incidence and aetiology of mastitis on dairy farms in England and Wales. The Veterinary Record, v.160, n.8, p.253-257, 2007.

- Britten, A. The role of diagnostic microbiology in mastitis control programs. The

- Veterinary Clinics of North America. Food Animal Practice, v.28, n.2, p.187-202, 2012.

- Cassoli, L.; Machado, P.; Codebella, A. Métodos de conservação de amostras de leite para determinação da contagem bacteriana total por citometria de fluxo. Revista Brasileira de Zootecnia, v.39, n.2, p.434-439, 2010.

- De Garnica, M.L.; Santos, J.; Gonzalo, C. Influence of storage and preservation on microbiological quality of silo ovine milk. Journal of Dairy Science, v.94, n.4, p.19221927, 2011.

- Elizondo, J.; Aldunate, A.; Ezcurra, P. et al. Efficiency of the proportion of azidiol on preservation in ewe’s milk samples for analysis. Food Control, v.18, n.3, p.185-190, 2007.

- Fu, Y.; Zhou, E.; Liu, Z. et al. Staphylococcus aureus and Escherichia coli elicit different innate immune responses from bovine mammary epithelial cells. Veterinary Immunology and Immunopathology, v.155, n.4, p.245-252, 2013.

- Kossaibati, M.; Esslemont, R. The costs of production diseases in dairy herds in England. Veterinary Journal, v.154, n.1, p.41-51, 1997.

- Leite, M. Fatores intereferentes na análise eletrônica da qualidade do leite cru conservado com azidiol liquido, azidiol comprimido e bronopol. Minas Gerais, Brasil: Universidade de Minas Gerais, 2006. Tese (Doutorado em Ciência Animal).

- Llopis, M.; Marugán, M.; Marugón, M. et al. Effect of storage and preservation of milk samples on the response of microbial inhibitor tests. The Journal of Dairy Research, v.80, n.4, p.475-484, 2013.

- Martins, M.; Nicolau, E.; Mesquita, A. et al. Bronopol and azidiol chemicals: time and temperature influence in the total bacterial count of raw milk. Ciência Animal Brasileira, v.10, n.2, p.627-633, 2009.

- National Mastitis Council. Microbiological procedures for the diagnosis of bovine udder infection and determination of milk quality. 4th edition. Verona, USA, 2004. 47p.

- Neder, V.; Signorini, M.; Cuatrin, A. et al. Prevalencia de bacterias patógenas de Mastitis bovina en leche de tanque de frío y evaluación de medios de cultivo para el 
recuento y la identificación de Staphylococcus aureus. Revista FAVE - Ciencias Veterinarias, v.13, n.1-2, p.21-28, 2014.

- Neidhardt, F.; Ingraham, J.; Schaechter, M. Physiology of the bacterial cell. A molecular approach. Sunderland, MA, USA: Sinauer associates, 1990. 507p.

- Olde, R.; Barkema, H.; Kelton, D. et al. Incidence rate of clinical mastitis on Canadian dairy farms. Journal of Dairy Science, v.91, n.4, p.1366-1377, 2008.

- Peixoto, R.; Araújo, R.; Peixoto, L. et al. Indirect diagnostic tests for the detection of subclinical mastitis in dairy goats experimentally infected with Staphylococcus aureus. Ciência Rural, v.46, n.7, p.1217-1222, 2016.

- Philpot, N.; Nickerson, S. Ganando la lucha contra la mastitis. 1.ed. Naperville, USA, 2000. 192p.

- Shim, E.; Shanks, R.; Morin, D. Milk loss and treatment costs associated with two treatment protocols for clinical mastitis in dairy cows. Journal of Dairy Science, v.87, n.8, p.2702-2708, 2004.

- Sierra, D.; Sánchez, A.; Contreras, A. et al. Short communication: effect of storage and preservation on total bacterial counts determined by automated flow cytometry in bulk tank goat milk. Journal of Dairy Science, v.92, n.10, p.4841-4845, 2009.

- Souza, G.; Faria, C.; Rios, R. Efeitos da temperatura e do tempo de armazenamento sobre a contagem total de bactérias em amostras de leite cru conservadas com azidiol. In: Congreso Nacional de Laticínios, 2006, Minas Gerais - Brasil. Memoria, v.61, p.358-361.

1 Financiado por la Unidad de Diagnóstico de la facultad de Ciencias Agrarias de la Universidad de Antioquia.

Cómo citar: Torres-Lindarte, G.; Vidal-Arboleda, J.; Caraballo-Guzmán, A.; VargasHoyos, K.; Olivera-Ángel, M. Efecto del conservante azidiol sobre la detección por cultivo microbiológico de bacterias causantes de mastitis bovina. Revista Veterinaria y Zootecnia, v.11, n.2, p.93-102, 2017. DOI: 10.17151/vetzo.2017.11.2.8

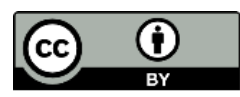

